\title{
REVIEW ON IOT BASED SMART GRID ARCHITECTURE IMPLEMENTATIONS
}

\author{
S. R. Mugunthan, \\ Associate Professor, Department of computer science and engineering, \\ Sriindu College of engineering and technology, Hyderabad, India. \\ Email id: srmugunth@gmail.com \\ Dr. T. Vijayakumar, \\ Professor, \\ Department of ECE, \\ Guru Nanak Institute of Technology, \\ Hyderabad, India. \\ Email: vishal_16278@yahoo.co.in
}

\begin{abstract}
There is a rapid development in Internet of Things and Smart Grid technologies in the recent days. In this paper, an extensive survey of Internet of Things (IoT) based Smart grid environments is done. These technologies, when used in combination offer energy optimization and user friendliness in terms on monitoring and controlling of electronic devices. The software solutions, challenges such as stability in connection, communication, cost and information privacy and security is also discussed broadly. This work exposes new perspectives and knowledge for researchers who work on interdisciplinary domains.
\end{abstract}

Keywords: IoT, Smart Grid, Data Security, Smart Buildings, Communication Technologies

\section{Introduction}

The distribution of digital power is increasing greatly day by day. The existing power grids are converted into smart grids so as to meet the growing power requirements. Information is accumulated from sensors, smart meters and several other devices for the sake of analysis and understanding. For the purpose of implementing IoT in smart grids, mobility support, location awareness, distributed coordination and latency sensitivity are to be considered. Smart Grid systems in combination with IoT can assist the consumption, distribution, transmission and generation of energy [3]. IoT allows smart monitoring and control of smart grid [8], [10].

In electronics connected via internet, smart plugs, home gateways and smart meters, application of IoT facilitates proficient resource management. The consumers can obtain information regarding consumption of energy and price on a real-time basis thereby moderate the energy consumption. The producer can forecast energy requirement and moderate distribution. Hence the system serves beneficial to both ends [7]. Millions of users 
Journal of Electrical Engineering and Automation (EEA) (2019)

Vol.01/ No. 01

Pages: $12-20$

https://www.irojournals.com/iroeea/

DOI: https://doi.org/10.36548/jeea.2019.1.002

interact with smart grids and its information flow. It is important to focus on scalability of this system. Cloud computing serves as an optimal solution for this purpose.

Several architectures such as event processing for load forecasting, lambda, kappa and cyclic architectures are designed and implemented for processing the data generated by these systems. In IoT based Smart Grid architectures, the components communicate with each other through the internet. Resource constraint and scarcity of spectrum are major issues in the wireless nodes of these systems [8]. Pan Wang et al [10] presented a programming model and fog based architecture that serves the requirements of smart grid. The paper also demonstrates its operation on a smart electric automobile prototype for the purpose of evaluation.

\section{Literature Review}

\section{A. Internet of Things}

Several elements and objects pertaining to a system are interlinked via IoT with the help of digital controllers, meters and sensors [4]. This interlinking allows remote control and monitor of the devices [17]. This system allows generation and accumulation of data via internet with zero human interaction [19]. Applying IoT in an energy based environment has led to the development of Internet of Energy (IoE). Several attacks occur while using IoT namely network, software, cryptanalysis, environmental, side-channel and physical attacks [18].

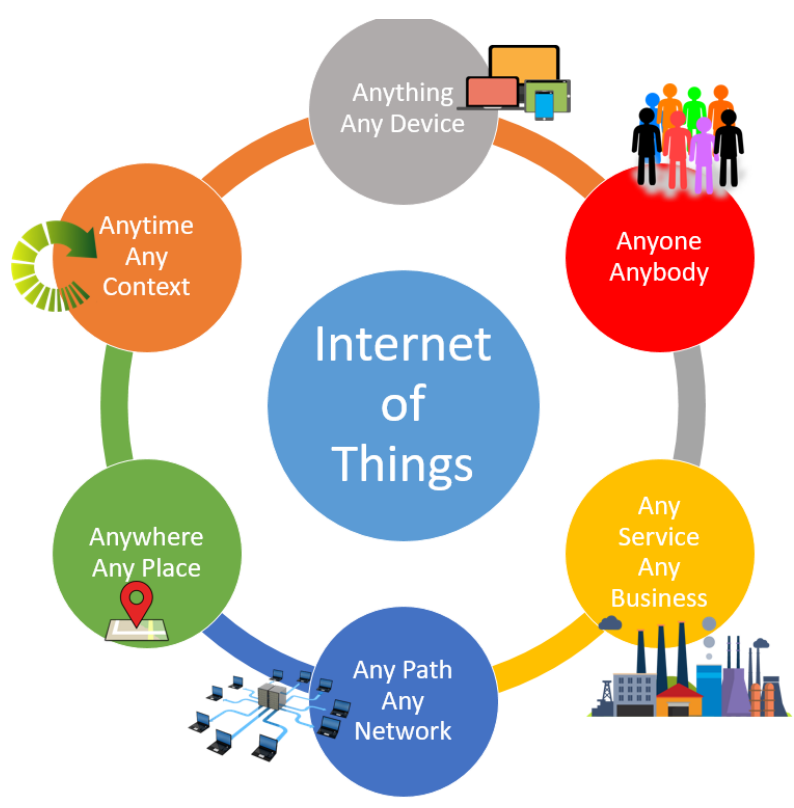

Figure 1: Internet of Things 
Journal of Electrical Engineering and Automation (EEA) (2019)

Vol.01/ No. 01

Pages: $12-20$

https://www.irojournals.com/iroeea/

DOI: https://doi.org/10.36548/jeea.2019.1.002

IoT is dependent on application programming interfaces (APIs) and several other technologies like cloud, machine learning, AI, predictive analytics, big data management and so on. The challenges and opportunities of IoT can be explored by enabling technologies, enhancing the connectivity models and realizing the transformational potentials.

\section{Smart Grid System}

The energy efficiency of smart grids helps optimization of buildings and other smart infrastructures. It allows data transfer along with the flow of current in both directions between grid and the building, hence providing real time feedback making the system more interactive and flexible [4]. Smart grid is a combination of digital communication along with electricity supply network. Smart grids are composed of Smart homes and buildings. Implementation of IoT in buildings increase the cost factor, but are still pursued as the benefits surpass the drawbacks.

In order to improve the efficiency while maintaining the energy requirements of the building, several innovative technologies and devices are invented. In traditional buildings, application of IoT leads to environmental safety, sustainability, comfort and increased efficiency. Smart grids provides the control to the consumers. It also benefits industries, institutions, retail stores and enterprises in tracking power consumption. Smart grids also have notable features like self-repair, ensuring leakage proof power supply of good quality, encouraging consumer to participate in the operations of the grid. 
Vol.01/ No. 01

Pages: $12-20$

https://www.irojournals.com/iroeea/

DOI: https://doi.org/10.36548/jeea.2019.1.002

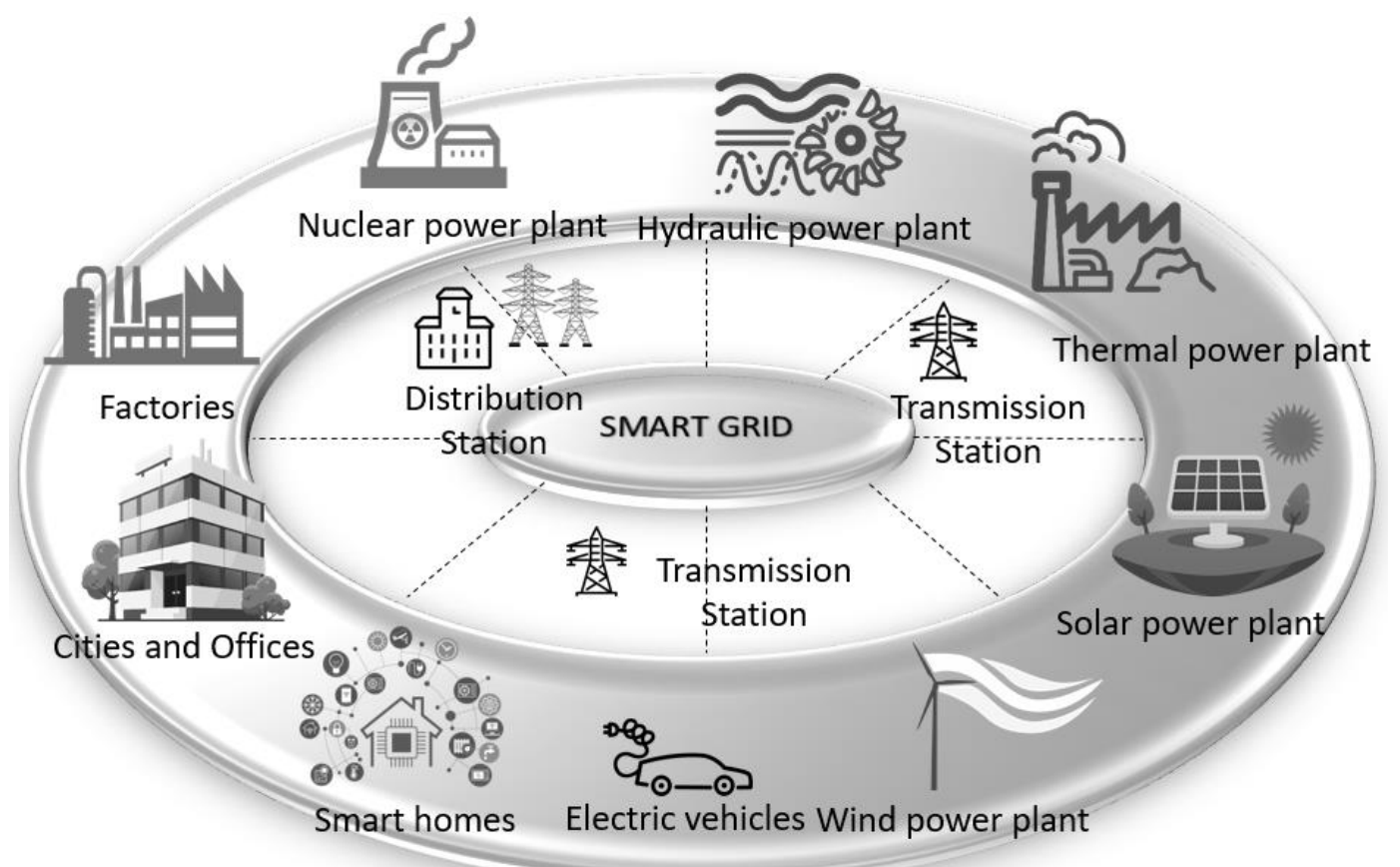

Figure 2: Architecture of Smart Grid

\section{B. IoT based Smart Grid Architectures}

Application of IoT in smart grids allows sharing of information between all components in the grid. In implementing smart cities with smart grids, IoT has a crucial role to play. IoT also enables smart energy management. Strong sensing capabilities and higher connectivity features of smart meters are exploited by IoT. The consumption patterns can be understood in large scale environments and can be used for optimization of energy and billing. Vasos Hadjioannou et al [11] worked on improving the privacy and security factors in the IoT based smart grids.

There are several challenges in implementing these systems. The major challenge is safety and privacy. It is essential to enforce safety in data collection, control messages, monitoring of equipment and notification transmission. Other factors to be considered include confidentiality, integrity, availability, authentication, nonrepudiation and access control. Several passive attacks like release of message contents and traffic analysis and active attacks like denial of service, jamming, False Data Injection (FDI) and masquerade are to be addressed. For this purpose, firewalls and encryption techniques are considered. Flocking-based model, warning systems, Jamming Attack Detection Based on Estimation (JADE), Cryptographic Keys, Real-Time Detection of False Data Injection, Minimizing Message Delay under Jamming Conditions, Aggregated Key Encryption, Intrusion Detection and such solutions are explored by several researchers. 
Vol.01/ No. 01

Pages: $12-20$

https://www.irojournals.com/iroeea/

DOI: https://doi.org/10.36548/jeea.2019.1.002

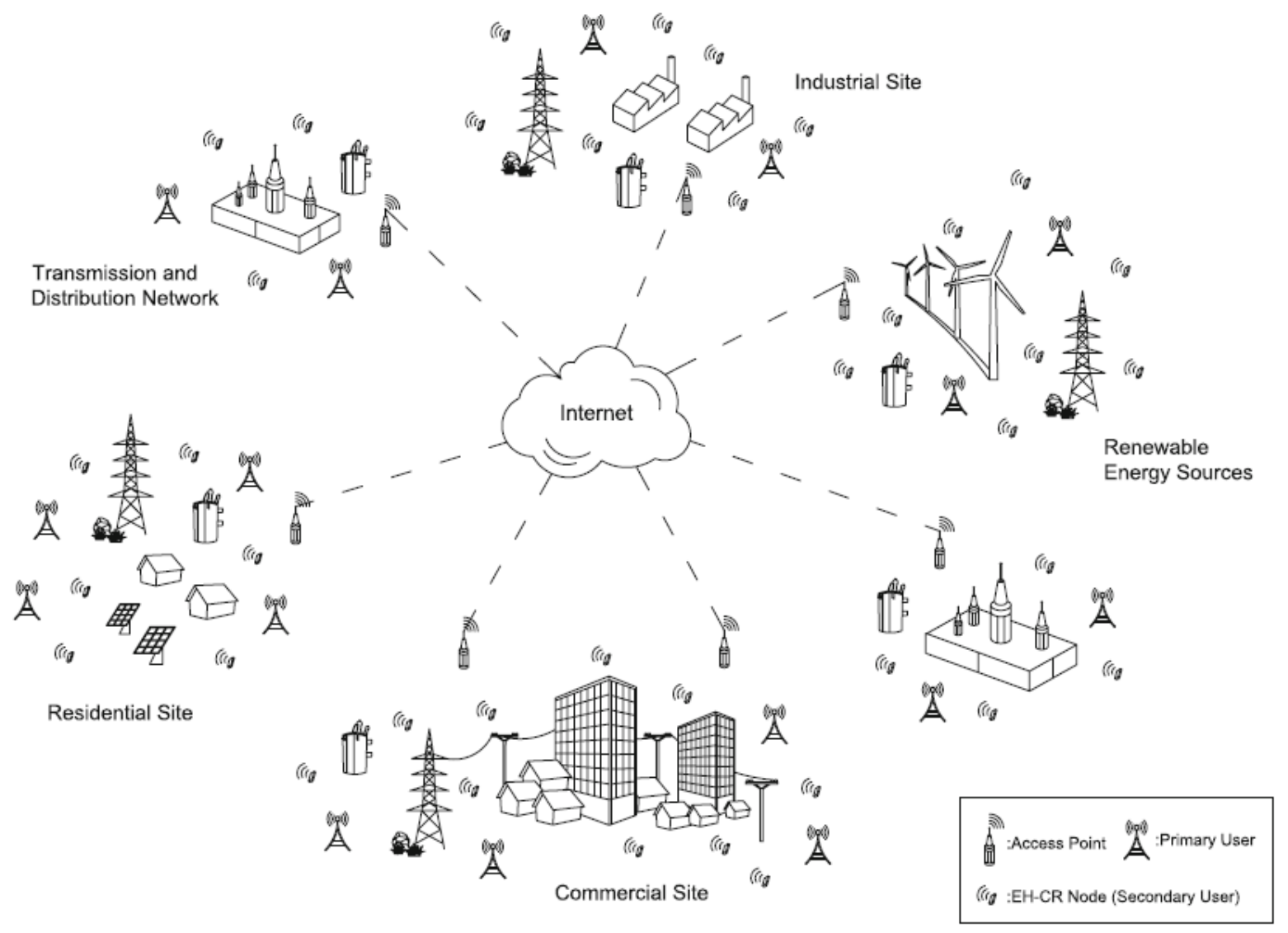

Figure 3: Smart Grid Architecture based on IoT [8]

\section{Challenges in Implementation of IoT based Smart Grids}

\section{A. Connection Stability and Communication}

The system response in smart grids reallocates the surplus power to the area where there is shortage in power locally. This improves the efficiency of the system. Similarly, it can work efficiently in several scenarios. It is essential to establish communication between systems. For proper communication, IoT needs fast internet connectivity. Low connectivity leads to reduction in optimality of redistribution of power. Small latency and quick response is a major requirement for smooth communication among the components in smart grid. Collection of scalable data is a major challenge due to the lack of tools for live analysis.

Also, during severe disasters, it is difficult to collect data and enable event logging hardware. It is also essential to implement cloud storage as the data will be streamed in large quantities while implementing the system in smart cities. 
Journal of Electrical Engineering and Automation (EEA) (2019)

Vol.01/ No. 01

Pages: $12-20$

https://www.irojournals.com/iroeea/

DOI: https://doi.org/10.36548/jeea.2019.1.002

\section{B. Cost}

It is simpler to establish a wireless network whereas, implementation of wired network involves proper planning and installation. While implementing smart grids, power failure are intolerable. This factor may lead to increased cost of implementation [13]. Despite the huge implementation costs, smart grids and IoT save large amounts of energy by rerouting power instantly on detection of power failure. Installation of smart meters are implemented in real time in France, USA and several countries along with the connection of several devices to IoT.

In certain application of IoT in smart grid, power distribution, transmission and distribution causes severe electromagnetic interference. To avoid this, the chips and equipment are to be made to withstand low and high temperature variations, with anti-vibration and anti-electromagnetic capabilities, enabled with water and dustproof systems and new technologies so as to improve the lifetime of the module.

\section{Information Security}

Smart grids and IoT are vulnerable to several security issues. Internet based security issues, cyber threats, resource constraints, data privacy, trust management, authorization and authentication, data integrity, cyberattacks, scalability, confidentiality and identity spoofing are some of the commonly faced issues. Security tools such as deep packet inspection and information management tools can provide security to a certain extent in IoT based smart grids. These techniques can address specific vulnerabilities and secure data threads. Wired networks are prone to physical damage and interruption. In case of wireless networks, despite strong encryption techniques, the data packets tend to be seized and decrypted in certain cases.

Cameras and sensors in smart environment can capture every movement and attract cybercriminals. Hence it is essential to create a secure environment and avoid leakage and misuse of data. Block chain and similar platforms can be used to ensure this security [1]. Poor design in the system may lead to vulnerabilities and bugs. It is difficult to integrate reliable and complex security methods due to the power constraints in IoT and smart meters [6]. The most frequent threats to information security includes remote controlling of smart meters, violation of confidentiality of consumers, alteration of energy transaction and grid destabilization, data monitoring for falsified reasons.

\section{Conclusion}


Journal of Electrical Engineering and Automation (EEA) (2019)

Vol.01/ No. 01

Pages: $12-20$

https://www.irojournals.com/iroeea/

DOI: https://doi.org/10.36548/jeea.2019.1.002

The combination of smart grid and IoT are discussed thoroughly with its components and software solutions. Smart city and home management with smart energy savings is also discussed. The challenges of the system such as privacy and security, connection stability, communication and cost are deliberated along with the prescription of some solutions to overcome the issues. The existing systems that integrate smart grids and internet of things are reviewed. The applications, architecture and vision of the systems are described. The network functions are improved at power utilization, distribution, transmission and generation.

The applications of the system includes power equipment installation surveillance, application of dynamic scheduling for adjustment of home consumption, parking and charging of electric vehicles, management of supply and demand of power, power supply equipment maintenance, detection of failure and faults and so on. Future work is focussed on addressing the challenges in each layer of architecture for factors like energy acquisition, communication, congestion, handling huge data, standardization, trust management, identity spoofing, scalability and so on.

\section{References}

[1] Swastika, Adi Candra, Resa Pramudita, and Rifqy Hakimi. "IoT-based smart grid system design for smart home." In 2017 3rd International Conference on Wireless and Telematics (ICWT), pp. 49-53. IEEE, 2017.

[2] Mocrii, Dragos, Yuxiang Chen, and Petr Musilek. "IoT-based smart homes: A review of system architecture, software, communications, privacy and security." Internet of Things 1 (2018): 81-98.

[3] Reka, S. Sofana, and Tomislav Dragicevic. "Future effectual role of energy delivery: A comprehensive review of Internet of Things and smart grid." Renewable and Sustainable Energy Reviews 91 (2018): 90108.

[4] Shahinzadeh, Hossein, Jalal Moradi, Gevork B. Gharehpetian, Hamed Nafisi, and Mehrdad Abedi. "IoT Architecture for Smart Grids." In 2019 International Conference on Protection and Automation of Power System (IPAPS), pp. 22-30. IEEE, 2019.

[5] Meloni, Alessio, Paolo Attilio Pegoraro, Luigi Atzori, Andrea Benigni, and Sara Sulis. "Cloud-based IoT solution for state estimation in smart grids: Exploiting virtualization and edge-intelligence technologies." Computer Networks 130 (2018): 156-165.

[6] Lombardi, Federico, Leonardo Aniello, Stefano De Angelis, Andrea Margheri, and Vladimiro Sassone. "A blockchain-based infrastructure for reliable and cost-effective IoT-aided smart grids." (2018): 42-6.

[7] Carvalho, Otávio, Eduardo Roloff, and Philippe OA Navaux. "A Distributed Stream Processing based Architecture for IoT Smart Grids Monitoring." In Companion Proceedings of the10th International Conference on Utility and Cloud Computing, pp. 9-14. ACM, 2017. 
Journal of Electrical Engineering and Automation (EEA) (2019)

Vol.01/ No. 01

Pages: 12-20

https://www.irojournals.com/iroeea/

DOI: https://doi.org/10.36548/jeea.2019.1.002

[8] Ozger, Mustafa, Oktay Cetinkaya, and Ozgur B. Akan. "Energy harvesting cognitive radio networking for IoT-enabled smart grid." Mobile Networks and Applications 23, no. 4 (2018): 956-966.

[9] Bhuiyan, Md Zakirul Alam, Mdaliuz Zaman, Guojun Wang, Tian Wang, Md Arafat Rahman, and Hai Tao. "Protected bidding against compromised information injection in IoT-based smart grid." In International Conference on Smart Grid and Internet of Things, pp. 78-84. Springer, Cham, 2018.

[10] Wang, Pan, Shidong Liu, Feng Ye, and Xuejiao Chen. "A fog-based architecture and programming model for iot applications in the smart grid." arXiv preprint arXiv:1804.01239 (2018).

[11] Hadjioannou, Vasos, Constandinos X. Mavromoustakis, George Mastorakis, Jordi Mongay Batalla, Ioannis Kopanakis, Emmanouil Perakakis, and Spiros Panagiotakis. "Security in smart grids and smart spaces for smooth IoT deployment in 5G." In Internet of Things (IoT) in 5G Mobile Technologies, pp. 371-397. Springer, Cham, 2016.

[12] Ghasempour, Alireza. "Optimum number of aggregators based on power consumption, cost, and network lifetime in advanced metering infrastructure architecture for Smart Grid Internet of Things." In 2016 13th IEEE Annual Consumer Communications \& Networking Conference (CCNC), pp. 295-296. IEEE, 2016.

[13] Tanwar, Sudeep, Sudhanshu Tyagi, and Sachin Kumar. "The role of internet of things and smart grid for the development of a smart city." In Intelligent Communication and Computational Technologies, pp. 23-33. Springer, Singapore, 2018.

[14] Hussain, Md, Mohammad Saad Alam, and M. M. Beg. "Fog computing in IoT aided smart grid transitionrequirements, prospects, status quos and challenges." arXiv preprint arXiv:1802.01818 (2018).

[15] Yun, Miao, and Bu Yuxin. "Research on the architecture and key technology of Internet of Things (IoT) applied on smart grid." In 2010 International Conference on Advances in Energy Engineering, pp. 6972. IEEE, 2010.

[16] Dalipi, Fisnik, and Sule Yildirim Yayilgan. "Security and privacy considerations for iot application on smart grids: Survey and research challenges." In 2016 IEEE 4th International Conference on Future Internet of Things and Cloud Workshops (FiCloudW), pp. 63-68. IEEE, 2016.

[17] Kumar, R. Praveen, and S. Smys. "A novel report on architecture, protocols and applications in Internet of Things (IoT)." In 2018 2nd International Conference on Inventive Systems and Control (ICISC), pp. 1156-1161. IEEE, 2018.

[18] Sridhar, S., and S. Smys. "Intelligent security framework for iot devices cryptography based end-to-end security architecture." In 2017 International Conference on Inventive Systems and Control (ICISC), pp. 1-5. IEEE, 2017.

[19] Suma, V. "Towards Sustainable Industrialization Using Big Data and Internet Of Things." Journal of ISMAC 1, no. 01 (2019).

[20] Li, Yuke, Xiang Cheng, Yang Cao, Dexin Wang, and Liuqing Yang. "Smart choice for the smart grid: Narrowband Internet of Things (NB-IoT)." IEEE Internet of Things Journal 5, no. 3 (2017): 1505-1515. 
Journal of Electrical Engineering and Automation (EEA) (2019)

Vol.01/ No. 01

Pages: $12-20$

https://www.irojournals.com/iroeea/

DOI: https://doi.org/10.36548/jeea.2019.1.002

[21] Persia, Samuela, Claudia Carciofi, and Manuel Faccioli. "NB-IoT and LoRA connectivity analysis for M2M/IoT smart grids applications." In 2017 AEIT International Annual Conference, pp. 1-6. IEEE, 2017.

[22] Arasteh, H., V. Hosseinnezhad, V. Loia, A. Tommasetti, O. Troisi, M. Shafie-Khah, and P. Siano. "Iotbased smart cities: a survey." In 2016 IEEE 16th International Conference on Environment and Electrical Engineering (EEEIC), pp. 1-6. IEEE, 2016.

[23] Mahmood, Khalid, Shehzad Ashraf Chaudhry, Husnain Naqvi, Taeshik Shon, and Hafiz Farooq Ahmad. "A lightweight message authentication scheme for Smart Grid communications in power sector." Computers \& Electrical Engineering 52 (2016): 114-124. 